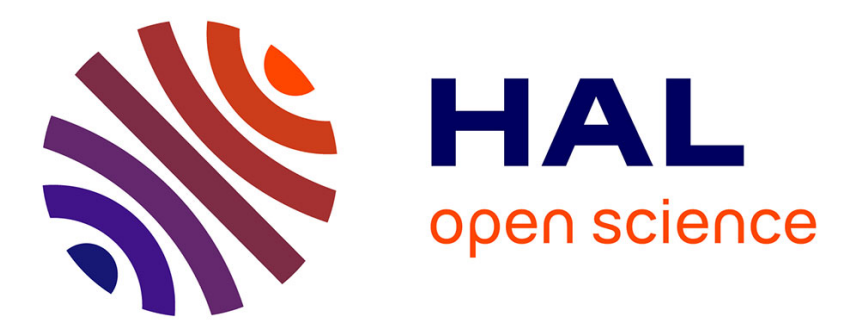

\title{
Phase Separation and Nanodomain Formation in Hybrid Polymer/Lipid Vesicles
}

T. P. Tuyen Dao, Fabio Fernandes, Meriem Er-Rafik, Romain Salva, Marc Schmutz, Annie Brûlet, Manuel Prieto, Olivier Sandre, Jean-François Le

Meins

\section{To cite this version:}

T. P. Tuyen Dao, Fabio Fernandes, Meriem Er-Rafik, Romain Salva, Marc Schmutz, et al.. Phase Separation and Nanodomain Formation in Hybrid Polymer/Lipid Vesicles. ACS Macro Letters, 2015, 4 (2), pp.182-186. 10.1021/mz500748f . hal-01383233

\section{HAL Id: hal-01383233 https://hal.science/hal-01383233}

Submitted on 30 Oct 2018

HAL is a multi-disciplinary open access archive for the deposit and dissemination of scientific research documents, whether they are published or not. The documents may come from teaching and research institutions in France or abroad, or from public or private research centers.
L'archive ouverte pluridisciplinaire HAL, est destinée au dépôt et à la diffusion de documents scientifiques de niveau recherche, publiés ou non, émanant des établissements d'enseignement et de recherche français ou étrangers, des laboratoires publics ou privés. 


\title{
Phase separation and nanodomain formation in hybrid polymer/lipid vesicles
}

\author{
T. P. Tuyen Dao ${ }^{\mathrm{a}, \mathrm{b}, \mathrm{c}}$, F. Fernandes ${ }^{\mathrm{c} *}$, M. Er-Rafik ${ }^{\mathrm{d}}$, R. Salva $^{\mathrm{a}, \mathrm{b}}$, M. Schmutz $^{\mathrm{d}}$, A. Brûlet $^{\mathrm{e}}$, M. Prieto $^{\mathrm{c}}$, \\ O. Sandre ${ }^{\mathrm{a}, \mathrm{b}}, \mathrm{J}-\mathrm{F}$. Le Meins ${ }^{\mathrm{a}, \mathrm{b}}$ * \\ a: University of Bordeaux, LCPO UMR 5629, 16 avenue Pey Berland, F-336oo Pessac, France \\ b: CNRS, Laboratoire de Chimie des Polymères Organiques, UMR 5629, F-3360o, Pessac, France \\ c: Centro de Química-Física Molecular and Institute of Nanoscience and Nanotechnology, Universidade de Lisboa \\ Instituto Superior Técnico, 1049-0o1 Lisboa, Portugal \\ d: Institut Charles Sadron, UPR 22 CNRS, Université de Strasbourg, 23 rue du Loess, 67034 Strasbourg, France \\ e: Laboratoire Léon Brillouin, UMR12 CEA-CNRS, CEA Saclay, F-91191 Gif-sur-Yvette Cedex, France
}

\begin{abstract}
Hybrid polymer/lipid large unilamellar vesicles (LUVs), were studied by small angle neutron scattering (SANS), time-resolved Förster resonance energy transfer (TR-FRET) and cryo-transmission electron microscopy (CryoTEM). For the first time in hybrid vesicles, evidence for phase separation at the nanoscale was obtained, leading to the formation of stable nanodomains enriched either in lipid or polymer. This stability was allowed by using vesicle-forming copolymer with a membrane thickness close to the lipid bilayer thickness, thereby minimizing the hydrophobic mismatch at the domain periphery. Hybrid giant unilamellar vesicles (GUVs) with the same composition have been previously shown to be unstable and susceptible to fission, suggesting a role of curvature in the stabilization of nanodomains in these structures.
\end{abstract}

Lipid nanodomains in biological membranes are thought to play a key role in several cell processes, ${ }^{1,2}$ but their small size (10-20onm) implies complex detection and characterization methodologies. Therefore many theoretical and experimental works ${ }^{3,4}$ were devoted to synthetic model membranes in order to unveil the salient features and the parameters governing the formation of such domains and the modulation of their size. Studies performed on multicomponent lipid vesicles showed that the fluidity of the different lipids and lipid-lipid interactions through their hydrophobic tails or polar head play important roles on phase separation and consequently, on domains formation. ${ }^{5}$ So far, the energy per unit length at the domain boundary (line tension) was shown to be of paramount importance. Bilayer thickness mismatch in multicomponent lipid vesicles modulates this line tension and domain size, as nicely evidenced by Heberle et al. using small angle neutron scattering (SANS) with contrast variation. ${ }^{6}$ In parallel, polymersomes obtained by selfassembly of amphiphilic block copolymers emerged in the late 9o's as an alternative to liposomes, e.g., in the field of drug delivery: the improved stability of their membrane compared to liposomes and the ease to modulate their functionality by coupling chemistry offers unprecedented new possibilities. ${ }^{7}$ The formation of domains in polymersome membrane was also investigated, in order to tune their membrane properties. ${ }^{8,9}$

Recently, hybrid polymer/lipid vesicles appeared as an ideal "upgrade" ${ }^{10}$ of their forerunners as they can marry in a single membrane the best characteristics of the two different systems (stability, bio-functionality, controlled permeability...). Promising results were observed regarding for instance drug targeting or bio-molecular recognition. ${ }^{11,12}$ To date, physical and molecular parameters governing the phase separation in these hybrid membranes are not well understood. In addition to the expected chemical incompatibility between polymer block chains and lipids, one has to consider also their dimensional differences. In order to perfectly exploit the potential of such structures, phase separation needs to be tuned, especially the way to formulate lipid nanodomains. As previously pointed out, the characterization of nanodomains in multicomponent vesicles is not straightforward as they cannot be observed directly by optical microscopy. Therefore only rather few results are available for lipid systems, ${ }^{6,13,14,15-18}$ and no evidence has yet been offered for hybrid polymer/lipid small or large vesicles. ${ }^{10}$ We seek to clarify the membrane structure of hybrid polymer vesicles and especially the way to obtain nanodomains enriched (or pure) either in lipids or in polymers. 


\begin{tabular}{|c|c|c|c|c|}
\hline & $\begin{array}{c}R_{\text {cyl }} \pm \sigma_{R}(\mathrm{~nm}) \text { or } \\
R_{\text {ves }} \pm \sigma_{R}(n m)^{*}\end{array}$ & $\begin{array}{c}R_{\mathrm{G}}(\mathrm{nm}) \\
(\text { Guinier } \\
\text { plot) }\end{array}$ & $\begin{array}{c}\text { Flat cylinder } L_{\text {cyl }} \pm \sigma_{\mathrm{L}}(\mathrm{nm}) \text { or } \\
\text { shell thickness } \delta(\mathrm{nm})^{*}\end{array}$ & $\begin{array}{c}\text { Membrane thickness }(\mathrm{nm}) \\
\text { (Kratky-Porod plot) }\end{array}$ \\
\hline Polymer matching $20^{\circ} \mathrm{C}$ & $54 \pm 9$ & 35 & $3.4 \pm 0.7$ & 3.4 \\
\hline Polymer matching $46^{\circ} \mathrm{C}$ & $73 \pm 7$ & 43 & $3.1 \pm 0.9$ & 3.3 \\
\hline No matching $20^{\circ} \mathrm{C}$ & $30 \pm 17^{*}$ & 52 & $5.1 \pm 1.2^{*}$ & 5.8 \\
\hline No matching $46^{\circ} \mathrm{C}$ & $37 \pm 14^{*}$ & 54 & $5.1 \pm 1.2^{*}$ & 5.6 \\
\hline
\end{tabular}

Table 1: Parameters obtained by either fitting the SANS curves from hybrid PDMS-g-(PEO) $/$ / DPPC ( $50 / 50$ mol. ratio) with flat cylinder (polymer matching) or vesicle* (no matching) form factor, and from Guinier and Kratky-Porod representations.

Although biophysical mechanisms which prevent ripening of small lipid domains in a membrane involve several parameters and are not yet completely understood, we decided to minimize line tension, which naturally arises from the thickness mismatch between lipid domain and the surrounding polymer membrane, since this parameter has been shown to play a role in the membrane structuration of giant hybrid vesicles. ${ }^{19,20}$ In this way, we selected a close to matching system, using a commercial grafted copolymer with a flexible poly(dimethylsiloxane) backbone and two poly(ethylene oxide) pendant moieties (PDMS- $g$ (PEO)2) of $M_{\mathrm{w}}=3000 \mathrm{~g} \cdot \mathrm{mol}^{-1}$, (see Supp. Info. for other details).

This copolymer is well known to form vesicles by itself with a membrane thickness $(\sim 5 \mathrm{~nm})$ close to that of liposomes $(\sim 3-4 \mathrm{~nm}){ }^{21,22}$ It was mixed with 1,2-dipalmitoyl-snglycero-3-phosphocholine (DPPC) or palmitoyl-oleoyl-snglycero-3-phosphocholine (POPC) at molar ratios of $50 / 50(\sim 80 / 20$ in polymer/lipid weight ratio). For the same composition in giant unilamellar vesicles (GUV), phase-separation with micrometer-sized lipid domains was observed by fluorescence microscopy. ${ }^{10,20}$ The present study was performed with lipid either in a fluid state (POPC at room temperature or DPPC at $46^{\circ} \mathrm{C}$ ) or in gel state (DPPC at room temperature). The vesicles produced (see Supp. Info. Section S2.2) were analyzed by dynamic and static light scattering (DLS / SLS). They possess a narrow size distribution and a hydrodynamic radius in agreement with the extrusion process (see Figure S-3). The hybrid character of the vesicles was checked by flow cytometry (FACS) and zeta potential measurements. (Supp. Info. Figure S-4, S-5, S-6).

The nanostructures formed were characterized by CryoTEM which has recently been used to characterize homogeneous hybrid polymer/lipid vesicles obtained from mixtures of another copolymer $\left(\mathrm{PDMS}_{60}-b-\mathrm{PMOXA}_{21}\right)$ with DPMC. ${ }^{23}$ Figure 1 is a representative micrograph of the two main morphologies observed: on the one hand rounded spherical vesicles, on the other hand faceted vesicles. The faceting is ascribed to the gel state of the DPPC phase. Similar pictures were indeed already obtained with pure DPPC vesicles in previous studies. ${ }^{24}$ (see also control in Supp. Info. S3.6) Rounded spherical vesicles are probably hybrid vesicles in which the lipid content is too small to see the faceting effect. It is important to note that the polymer/lipid composition is not perfect- ly controlled within the vesicle population as vesicles are known to be out-of-equilibrium objects ${ }^{25-27}$

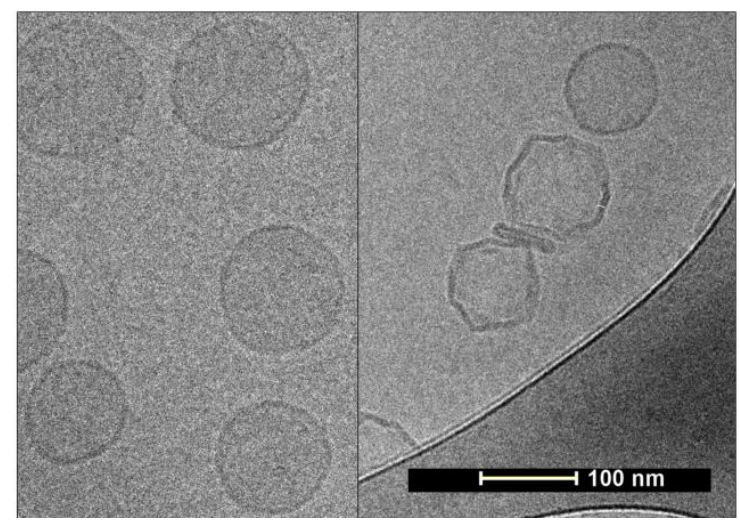

Figure 1: Cryo-TEM pictures of 8o/20 weight ratio PDMS- $g$ $(\mathrm{PEO})_{2} / \mathrm{DPPC}$ vesicles, quenched from room temperature

To get more information about the membrane structure of LUV hybrid vesicles, we performed SANS experiments using $\mathrm{d}_{62}$-DPPC, i.e. DPPC with fully deuterated fatty chains. To separate the contribution to the scattering of the lipid and of the copolymer components, $\mathrm{D}_{2} \mathrm{O} / \mathrm{H}_{2} \mathrm{O}$ mixtures were used (Supp. Info. S3.4). LUV hybrid vesicles were also prepared in $\mathrm{D}_{2} \mathrm{O}$ with classical DPPC. Thus the solvent mixture matching the polymer enables the observation the lipid phase, while the lipid-matching solvent allows seeing the copolymer. Without matching, we can observe the whole hybrid vesicles. Such results are illustrated on Figure 2. Interestingly, the curves obtained in polymer matching condition could not be fitted with the vesicle form factor commonly used to model pure lipid or polymer vesicles, but they were well fitted with a polydisperse flat cylinder (disk-like) form factor (Supp. Info., S3.4). The fitting values of radius $\left(R_{\text {cyl }}\right)$ and height $(L)$ indicated in Table 1 suggest that lipid phase presents disk-like shapes, both at $20^{\circ} \mathrm{C}$ and $46^{\circ} \mathrm{C}$, whose thicknesses well correspond to the one of a pure lipid bilayer. Radius of gyration $\left(R_{\mathrm{G}}\right)$ and membrane thicknesses $(\delta)$ were also estimated using Guinier and Kratky-Porod plots, respectively (see Supp. Info.). Disk radii calculated from the independently measured values of $R_{\mathrm{G}}$ and $\delta$ through the equation $R_{\mathrm{G}}{ }^{2}=R_{\mathrm{cyl}}{ }^{2} / 2+\delta^{2} / 12$ are in good agreement with disk radii obtained using the disk-like form factor fit. It is interesting to note that the lipid/polymer volume ratio estimated geometrically is $\pi R_{\text {cyl }}{ }^{2} \times L_{\text {cyl }} /\left(4 \pi R_{\mathrm{G}}{ }^{2} \times \delta\right)$ $=0.18$ at $20^{\circ} \mathrm{C}$ and 0.28 at $46^{\circ} \mathrm{C}$, thus not differing too 
much from the mass ratio of 0.20 calculated from the composition and the molar masses. In lipid matching condition, data could be precisely fitted with neither a cylinder nor a vesicle form factor either (Supp. Info., S3.4). The curves would probably be fitted by a holey shell form factor that needs to be computed numerically in further studies.

In pure $\mathrm{D}_{2} \mathrm{O}$ (no matching), the data are very well fitted with a vesicle form factor as shown on Figure 2: the characteristic parameters are indicated in Table 1 , as well as estimates of $R_{\mathrm{G}}$ and $\delta$. The values are in agreement with the size of the vesicles expected from the extrusion process $(R \sim 50 \mathrm{~nm})$ and with membrane thicknesses reported previously for $\mathrm{DPPC}^{28}$ and for the copolymer. ${ }^{22,29}$

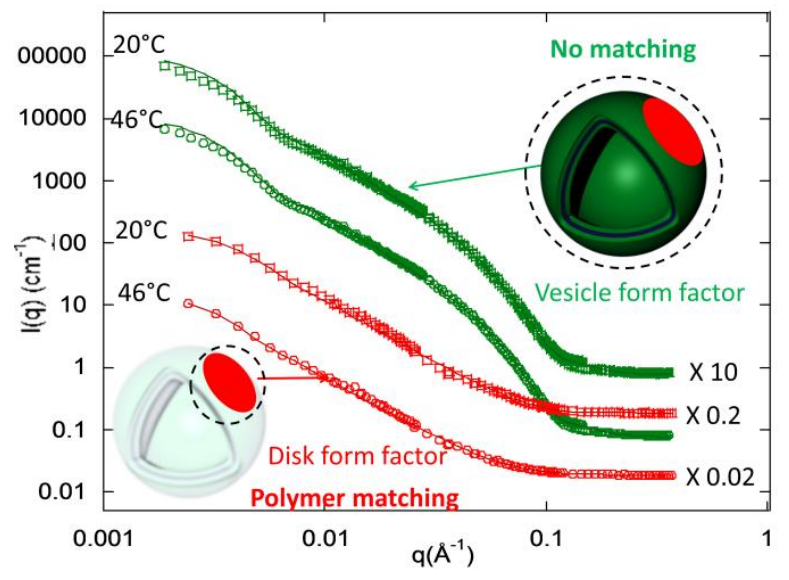

Figure 2: SANS data of PDMS- $g$ - $(\mathrm{PEO})_{2} / \mathrm{d}_{62}-\mathrm{DPPC}(8 \mathrm{o} / 20 \mathrm{wt}$. ratio) hybrid vesicles at $20^{\circ} \mathrm{C}$ and $46^{\circ} \mathrm{C}$ in polymer matching and no matching conditions. Solid lines: fitting curves.

This set of results proves that hybrid vesicles are obtained and that phase separation occurs within the membrane, leading to the formation of lipid domains detected as disks by SANS, floating in the surrounding polymer membrane. It also appears that phase separation is still present above the melting transition of DPPC, although the characteristic values of disk radii seem to be a little too large compared to the measured radii of gyration of the vesicles $\left(R_{\mathrm{G}} \sim 56 \mathrm{~nm}\right.$ by SLS and $R_{\mathrm{G}} \sim 52 \mathrm{~nm}$ by SANS in no matching condition), especially at $46^{\circ} \mathrm{C}$. As previously mentioned, polymer/lipid composition is not perfectly controlled from a vesicle to another and that probably leads to higher dispersity in disk sizes and to the existence of a population with lower lipid contents. Also, we insist of the point that the term "disk" is abusive for these lipid domains surrounded by polymer membrane as their shape may not be circular, especially in gel state and should follow the convex curvature of the vesicle, in fluid state. These aspects are not taken into account in our simple fitting procedure, which however correctly describes the phase separation phenomenon. The parameter that is measured the most precisely is the membrane thickness, whose values are close to the expected ones. A more precise estimate of the lipid domain size and shape by small angle neutron scattering deserves to be undergone in the future by a comprehensive study on a larger range of compositions in a higher flux neutron reactor.
To get more insight into the membrane structure of these hybrid vesicles, and especially to probe demixing at the nanometer scale between the lipid and polymer phases, we used Förster resonance energy transfer (FRET). ${ }^{17}$ This is a powerful method to detect and characterize lateral membrane domains presenting sizes smaller than 50-100 $\mathrm{nm}$. PDMS- $g$-(PEO) $)_{2}$ tagged with the NBD molecule and $\mathrm{N}$-(lissamineRhodamineBsulfonyl)-1,2-dioleoylsn-3-phosphatidylehanolamine (Rhod-PE) were used respectively as the donor and acceptor pair (See S3.5 of Supp. Info. for experimental procedure and data analysis). At first, the phase separation within the membrane was proven by measuring the partition coefficient of the probes, PDMS- $g$-(PEO) $)_{2}$-NBD and Rhod-PE in both DPPC and POPC with PDMS- $g$-(PEO) $)_{2}$ hybrid vesicles when the lipid was in its fluid state (Supp. Info. S3.5.1).

The PDMS- $g$-(PEO) $)_{2}$-NBD probe partitions almost exclusively in the polymer phase, which implicitly demonstrates phase separation between polymer and lipid. We also observed that the labeled lipid incorporates in the polymer phase to some extent (39 and $37 \mathrm{~mol} \%$ ) for equimolar PDMS- $g$-(PEO) $)_{2} / \mathrm{DPPC}$ and PDMS- $g$ $(\mathrm{PEO})_{2} / \mathrm{POPC}$ mixtures, respectively. (See Supp info. S3.5.1.)

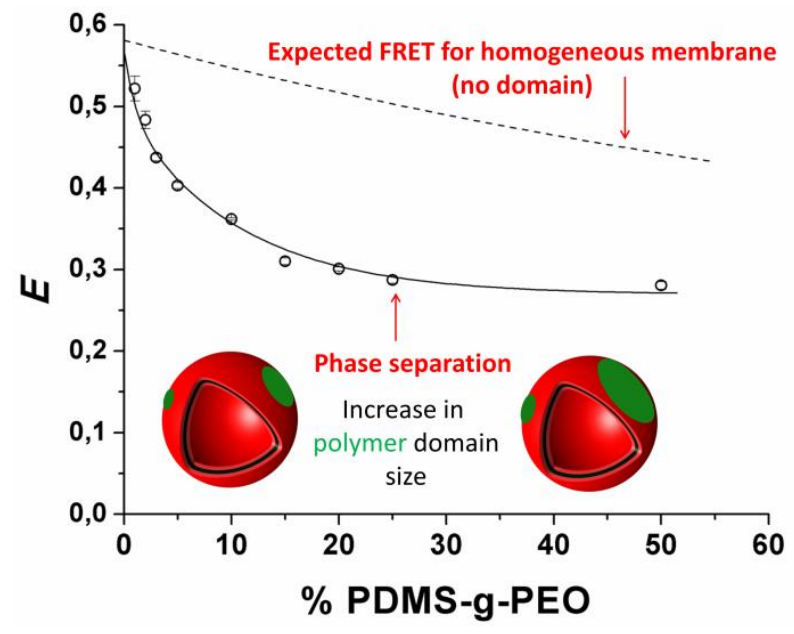

Figure 3: FRET efficiency versus content (mol \%) PDMS- $g$ $(\mathrm{PEO})_{2}$. Solid line is a guide to the eyes.

The phase separation was further proven by comparing the experimental FRET efficiency in PDMS- $g$-(PEO) $)_{2}^{-}$ NBD/POPC/DOPE-Rho (1/98.5/o.5 mol $/ \mathrm{mol} / \mathrm{mol})$ mixed vesicles (Supp. Info., S3.5.3) with the theoretical expectation value (formalisms described in Supp. Info. S3.5.2). FRET efficiencies were measured at different polymer/lipid ratios (Figure 3). The significant decrease in FRET efficiencies observed for increasing molar fractions of polymer is consistent with the increase of polymer domain sizes, and therefore increase of the average distance between labeled PDMS- $g$-(PEO) $)_{2}-\mathrm{NBD}$ donor and Rhod-PE acceptor. In case of a homogeneous lipidpolymer membrane, the predicted decrease in FRET efficiency upon increase of PDMS- $g$-(PEO) $)_{2}$ molar fraction would be much smaller (dotted line in Figure 3). 
In the following, we prove that the plateau observed at PDMS- $g-(\mathrm{PEO})_{2} / \mathrm{PC}$ proportion higher than $25 \mathrm{~mol} \%$ is due to the formation of polymer-rich domains larger that 5-10 times $R_{0}$, the Förster distance for this donor-acceptor pair, i.e. about 25-50 nm. (See Supp. Info. S3.5.2 and S3.5.4 for details). The acceptor Rhod-PE lipid is still present in these polymer raft-like domains, as already quantified through its partition coefficient between the lipid and polymer phases and reported in the preceding paragraph. We measured the FRET efficiency within the PDMS- $g$ $(\mathrm{PEO})_{2}$-rich phase of the 50/50 PDMS-g-(PEO)2/DPPC mixture with different concentrations of the acceptor Rhod-PE.

The recovered experimental FRET efficiencies are in perfect agreement with the FRET values calculated with acceptor concentrations obtained from the partition coefficient experiments and well below those expected for a homogeneous membrane. This demonstrates that there exists a negligible percentage of bordering PDMS- $g$ (PEO)2-NBD donor molecules transferring to acceptors in the DPPC-rich phase and provides good evidence for diameters of PDMS-g-(PEO)2 domains larger than $25-50$ $\mathrm{nm}$ in these hybrid polymer/ lipid vesicles. The same experiment was conducted on a 50/50 POPC/PDMS- $g$ $(\mathrm{PEO})_{2}$ mixture with comparable results (Figure 4 ).

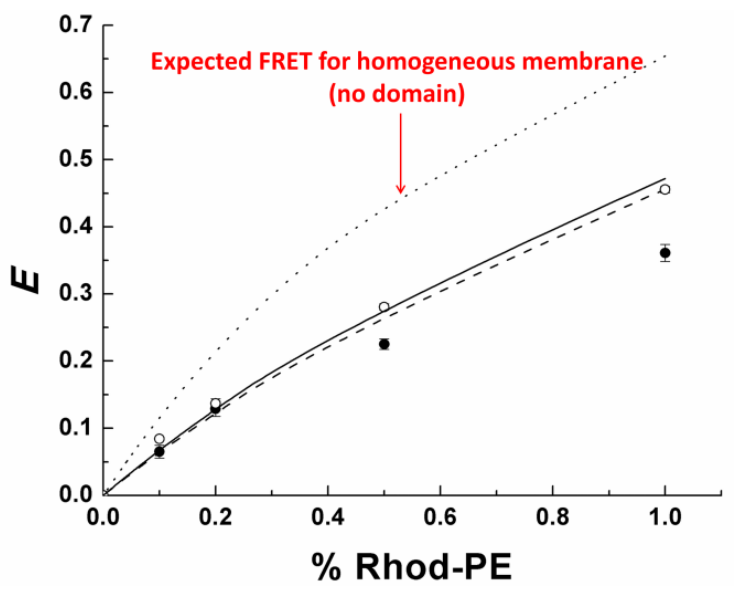

Figure 4: FRET efficiencies versus content (mol \%) of acceptor for DPPC at $46^{\circ} \mathrm{C}(\mathrm{O})$ and for POPC at $25^{\circ} \mathrm{C}(\bullet)$. Theoretical values for FRET (Supp. Info.) with DPPC (-) or POPC (--) .

In conclusion, this work evidenced from neutron scattering and FRET data that a nanoscale phase separation is obtained in hybrid polymer/lipid LUV, leading to the formation of stable nanodomains enriched in either lipid or polymer, coexisting in the same patchwork membrane of the vesicles.

These nanodomains, whose exact shape and size remain to be determined, are observed whatever the fluid or gel state of the lipid. We hypothesize that the use of a flexible copolymer, well known to form polymersomes with a membrane thickness close to that of liposomes, decreases the thickness mismatch and allows a conformational adaptation of the polymer chains at the boundaries, decreasing the line tension and stabilizing the nanodomains, even in the fluid state. This thickness mismatch is of paramount importance for membrane structuration and control of domain sizes, as shown in the literature for membranes made by mixing lipids of various chain lengths. ${ }^{6,30}$

Interestingly, phase-separation inside these hybrid/polymer lipid vesicles was previously observed (through stable micrometric lipid domains) for giant vesicles obtained from the same polymer/lipid composition with lipid in a gel state ${ }^{20}$. However, with lipid in a fluid state for such composition, budding and fission phenomenon was observed after a few hours, leading to the formation of "pure" liposomes and polymersomes. There is no evidence of such a phenomenon in our experiments for LUVs. This suggests that membrane curvature could play a role also in the stabilization of nanodomains. A complete study in parallel on GUV, LUV and SUV vesicles, involving several block copolymers with different molar masses and a large compositional range is currently in progress to gain more insight into the parameters governing the phase separation and the formation of nanodomains in hybrid polymer/lipid vesicles.

This study is a first important step to elucidate the properties of these new self-assembled hybrid structures, and to allow their optimization regarding different fields of application, namely the design of drug delivery nanosystems with particulate targeting capability (through the possibility of bio-functionalization of the lipid and/or the polymer nanodomains), or of biomimetic experiments aimed at reproducing for instance focal adhesion points of biological cells.

\section{ASSOCIATED CONTENT}

\section{Supporting Information}

Structure of copolymer used, chemical modification procedures, light and neutrons scattering experiments, Cryo-TEM, zeta potential, flow cytometry, FRET experiments and analysis. This material is available free of charge via the Internet at http://pubs.acs.org."

\section{AUTHOR INFORMATION}

\section{Corresponding Authors}

lemeins@enscbp.fr

fernandesf@ist.utl.pt

Notes

The authors declare no competing financial interests.

\section{ACKNOWLEDGMENT}

H. De Oliveira and R. Siadous (U1026 INSERM) for their help in flow cytometry measurements. A. Fedorov for assistance in FRET measurements. Fundings: International doctoral school on Functional Materials (IDS-FunMat), Erasmus Mundus (EU), FCT (RECI/CTM-POL/0342/2012) and Agence Nationale de la Recherche (KbT 12-BS08-0018-01). F.F. acknowledges the support of Fundação para a Ciência e Tecnologia (FCT) via the SFRH/BPD/64320/2009 grant. 


\section{REFERENCES}

(1) Michel, V.; Bakovic, M. Biol Cell 2007, 99, 129.

(2) Anderson, R. G.; Jacobson, K. Science 2002, 296,

1821.

(3) Kuzmin, P. I.; Akimov, S. A.; Chizmadzhev, Y. A.; Zimmerberg, J.; Cohen, F. S. Biophys J 2005, 88, 1120.

(4) Elson, E. L.; Fried, E.; Dolbow, J. E.; Genin, G. M. Annu Rev Biophys 2010, 39, 207.

(5) Binder, W. H.; Barragan, V.; Menger, F. M. Angew. Chem. Int. Ed. 2003, 42, 5802.

(6) Heberle, F. A.; Petruzielo, R. S.; Pan, J.; Drazba, P.; Kucerka, N.; Standaert, R. F.; Feigenson, G. W.; Katsaras, J. J. Am. Chem. Soc. 2013, 1356853.

(7) Discher, D. E.; Eisenberg, A. Science 2002, 297,

967.

(8) Lopresti, C.; Massignani, M.; Fernyhough, C.; Blanazs, A.; Ryan, A. J.; Madsen, J.; Warren, N. J.; Armes, S. P.; Lewis, A. L.; Chirasatitsin, S.; Engler, A. J.; Battaglia, G. ACS nano 2011, 5, 1775 .

(9) Christian, D. A.; Tian, A.; Ellenbroek, W. G.; Levental, I.; Rajagopal, K.; Janmey, P. A.; Liu, A.; Baumgart, T.; Discher, D. E. Nature Mater. 2009, 8, 843.

(10) Le Meins, J. F.; Schatz, C.; Lecommandoux, S.; Sandre, O. Materials Today 2013, 16, 397.

(11) Schulz, M.; Werner, S.; Bacia, K.; Binder, W. H. Angew Chem Int Ed Engl 2013, 52, 1829.

(12) Cheng, Z.; Elias, D. R.; Kamat, N. P.; Johnston, E.; Poloukhtine, A. A.; Popik, V. V.; Hammer, D. A.; Tsourkas, A. Bioconjugate Chem. 2011, 22, 2021.

(13) Stefl, M.; Sachl, R.; Humpolickova, J.; Cebecauer, M.; Machan, R.; Kolarova, M.; Johansson, L. B.; Hof, M. Biophys J 2012 102, 2104.

(14) Sachl, R.; Humpolickova, J.; Stefl, M.; Johansson, L. B.; Hof, M. Biophys J 2011, 101, L60.

(15) de Almeida, R. F.; Loura, L. M.; Fedorov, A.; Prieto, M. J Mol Biol 2005, 346, 1109.
(16) Suga, K.; Umakoshi, H. Langmuir 2013, 29, 4830.

(17) Loura, L. M. S.; Fernandes, F.; Prieto, M. European Biophysics Journal with Biophysics Letters 2010, 39, 589.

(18) Brown, A. C.; Towles, K. B.; Wrenn, S. P. Langmuir 2007, 23, 11188

(19) Schulz, M.; Olubummo, A.; Bacia, K.; Binder, W. H. Soft Matter 2014, 10, 831.

(20) Chemin, M.; Brun, P. M.; Lecommandoux, S.; Sandre, O.; Le Meins, J. F. Soft Matter 2012, 8, 2867.

(21) Lin, Z.; Hill, R. M.; Davis, H. T.; Scriven, L. E.; Talmon, Y. Langmuir 1994, 10, 1008.

(22) Salva, R.; Le Meins, J.-F.; Sandre, O.; Brûlet, A.; Schmutz, M.; Guenoun, P.; Lecommandoux, S. ACS nano 2013, 7, 9298.

(23) Winzen, S.; Bernhardt, M.; Schaeffel, D.; Koch, A.; Kappl, M.; Koynov, K.; Landfester, K.; Kroeger, A. Soft Matter 2013, 9, 5883.

(24) Andersson, M.; Hammarström, L.; Edwards, K. Journal of Physical Chemistry 1995, 99, 14531.

(25) Döbereiner, H. G. Current Opinion in Colloid and Interface Science 2000, 5, 256.

(26) Jung, H. T.; Coldren, B.; Zasadzinski, J. A.; Iampietro, D. J.; Kaler, E. W. Proceedings of the National Academy of Sciences of the United States of America 2001, 98, 1353.

(27) Luo, L.; Eisenberg, A. Langmuir 2001, 17, 6804.

(28) Kučerka, N.; Nieh, M. P.; Katsaras, J. Biochimica et Biophysica Acta 2011, 1808, 2761.

(29) Carlsen, A.; Glaser, N.; Le Meins, J. F.; Lecommandoux, S. Langmuir 2011, 27, 4884.

(30) Garcia-Saez, A. J.; Chiantia, S.; Schwille, P. J Biol Chem 2007, 282, 33537. 
